\title{
Editorial: Pediatric Radiology for the Practitioners - Simplifying the Jargons
}

\author{
Arun K. Gupta ${ }^{1} \cdot$ Manohar Shroff $^{2}$
}

Received: 1 April 2016/Accepted: 1 April 2016/Published online: 25 April 2016

(C) Dr. K C Chaudhuri Foundation 2016

Medicine is a dynamic subject. Pediatric Medicine, like other medical specialties, has experienced major expansion in the past decades. Keeping in pace with the technological advancement and expansion in pediatric medicine; a growing demand of this era is to translate the advances in medical technology to useful imaging advancements. And understandably enough, pediatric radiology has evolved very rapidly in the recent past. So much so that pediatric radiology has already established itself as a separate recognised sub-specialty in itself. The oftused phrase 'children are not small adults' can only emphasize and reflect the importance of this subspecialty. Whereas basic radiological interpretation in children is a skill which is probably essential for a practicing pediatrician; the newer advanced imaging modalities (such as CT, MRI, CT and MR angiography) are best interpreted by a pediatric Radiologist. As a pediatric Radiologist, one has to be well trained with basic radiology as well as diseases specific to children; and be well versed with the technicalities of various procedures. Being extremely careful about the radiation issues is of major importance in pediatric imaging.

Pediatric Radiology, unfortunately, is not a wellestablished subspecialty in Indian scenario. Structured teaching program and fellowship in pediatric radiology is conducted in only a few central institutes in the country. Therefore, a basic teaching program and generation

Arun K. Gupta

arunk676@gmail.com

1 Department of Radio-Diagnosis, All India Institute of Medical Sciences, New Delhi 110029, India

2 Department of Pediatric Neuroimaging, Hospital for Sick Children, Toronto, Ontario, Canada of awareness about the importance of this subspecialty is the need of the hour. It is therefore a responsible and welcome step by The Indian Journal of Pediatrics to bring out a symposium on pediatric imaging in three issues (June, July and September 2016). Since detailed description of the entire pediatric imaging is beyond the scope of any periodicals; the topics will be divided into three modality-based broad divisions. In the June issue, the focus will be kept on the utility of radiographs and ultrasound in pediatric medicine. CT and interventions will be discussed in the July issue and MRI in the September number.

Plain radiographs are the most commonly performed imaging in any clinical practice. The radiographs are often interpreted by the practitioners themselves; especially so in an acute ICU setting or a busy OPD. Amongst all the investigations, Chest radiograph is the single most commonly performed imaging and its importance can not be undermined. A pediatric resident should have adequate skill to interpret a chest radiograph as abnormal and characterize the abnormality; which is often a critical part of the clinical decision-making. Hence, the first chapter in the June issue has a chapter on an approach to chest radiography, by Bhalla AS and colleagues. The authors have a vast experience in pediatric and thoracic radiology and have translated that into a lucid and informative text about pattern approach in chest radiograph [1]. Abnormality in a chest radiograph should be classified in specific patterns such as consolidation, mass, nodule, cysts etc. in order to reach a reasonable differential diagnosis. Musculoskeletal radiographs in children are also commonly performed for various indications; skeletal dysplasia being one major indication. While this area remains largely unexplored by both the pediatricians as well as radiologists; basic knowledge and a systematic approach towards the radiologic interpretation can yield truly gratifying results; leading the 
pediatrician and the radiologist towards a reasonable set of differential diagnoses. Dr Vijayalakshmi and colleagues have made the complex topic of skeletal dysplasia appear much simpler in their article [2] in the June issue of the symposium. The approach to diagnose any suspected skeletal dysplasia should follow a systematic way; with due importance to individual radiographs. Pelvic radiograph and spine radiograph are of immense importance; and can give a host of information, and often are sufficient to reach a diagnosis.

Ultrasonography as an imaging technique has shown major advancements in the form of advanced high resolution transducers suitable for pediatric imaging; 3D and 4D ultrasonography and superior color Doppler imaging. Being a safe imaging technique without any radiation burden, it remains to be the imaging modality of choice in the abdominal conditions in children; reserving the use of $\mathrm{CT}$ as a problem solving tool. In the June issue, Dr Saxena and colleagues have described the usefulness of abdominal sonography in children in great detail [3]. There is a precise and informative description of the imaging findings of all prevalent disease entities supplemented by good quality images. This article certainly can claim to be a ready guide to the radiologist as well as the pediatricians to be aware of all the disease conditions prevalent in children.

A brilliant example of the utility of technological advancements in ultrasonography is its application in pediatric small part sonography, especially musculoskeletal sonography. The detail with which the smaller tendons and cartilages are visualized with the present-day high frequency transducers was hitherto beyond imagination. This calls for a priming of the pediatricians towards the usefulness of musculoskeletal sonography in children; and the radiologists about the sonographic appearance of the abnormalities in musculoskeletal ultrasound. Pediatric brain imaging using cranial sonography can be performed through the anterior fontanel in younger infants. A specialized sector transducer with a small footprint can enable the radiologist to get a good quality scan. As with any other pediatric imaging, craniospinal imaging also needs expertise to perform and interpret the scans. Occult spinal dysraphism is an important indication of pediatric spinal sonogram (the AIUM guidelines) [4]. Although MRI is commonly performed in spinal dysraphism; spinal sonography can provide images with very good resolution and has the added advantage of visualizing the movements of the spinal nerve roots in real time which can rule out nerve root tethering. In the June issue of the symposium, Dr Karnik and collegaues have discussed in detail the role of ultrasonography in pediatric cranio-spinal and musculoskeletal diseases [5]. Non-accidental injury, contrary to the popular belief, is not uncommon in Indian scenario. The imaging maniferstations are varied; and the pediatrician and the radiologist both should have a high index of suspicion.
Also, being aware of the imaging findings in nonaccidental injury is crucial so as to avoid grave consequences. The June issue deals with a dedicated article on the imaging on non accidental injury (NAI) [6].

The July issue of the symposium has two articles by Dr Gibikote et al. on CT; one deals with the use of CT in pediatric chest conditions; and the other in pediatric abdomen [7, 8]. In pediatric chest conditions, CT is the most commonly used imaging modality after a chest radiograph. The utility of CT in recurrent or non-resolving infections, interstitial lung diseases, airway and suspected vascular problems can not be overemphasized. Newer multidetector row CT scanners are capable of imaging the whole chest in only a few seconds, thereby obviating the need for long breath hold/ prolonged sedation or anesthesia. This advantage, coupled with the advantage of superior spatial resolution and multiplanar reformats; make CT scan the investigation of choice in most of the pediatric thoracic conditions after an abnormal chest radiograph. Similarly, after an abnormal or inconclusive USG abdomen, CECT often remains the mainstay of imaging in pediatric abdomen. MRI in pediatric age group suffer from the disadvantages of prolonged scan time, susceptibility to various artifacts and the need for general anesthesia. With the advances in MR technology, pediatric body MRI has come of age and is on its way to establish itself as a dominant imaging modality free of radiation hazards. Superior MR coils, faster imaging sequences and higher field strength of the magnets have all contributed to this advancement. An introduction to this complex topic has been laid out in a very lucid manner by Dr Sharma in the September issue of the symposium [9]. The author has a vast experience in body MR imaging and has made a successful effort in laying out a very useful primer to pediatric body MR imaging both for the pediatrician as well as the radiologist.

Interventional radiology is not a myth anymore; and the scope of pediatric interventional radiology is ever-expanding. While this is a domain less travelled by the radiologists, proper training and practice in this field is required in the current era; in order to develop a team approach and multispecialty treatment of various diseases. Pediatric interventional radiology can broadly be divided into vascular and non-vascular interventions. Vascular interventions can range from chemoembolization of tumors, embolization of vascular malformations, to more emergent management of endovascular treatment of acute conditions like hemoptysis, hemobilia, hematuria and many more. Non-vascular interventions include various abscess and collection drainages, biopsies, drainage of empyema/ hydropneumothorax, percutaneous nephrostomy and stenting and special procedures like radiofrequency ablation of various tumors. While a detailed description is beyond the scope of the journal, the July issue of the symposium has two chapters dedicated to the pediatric interventional radiology, contributed by Gamanagatti et al. 
$[10,11]$. The author has vast experience in this domain and is the ideal person to describe the nuances of the procedures, in a much simplified manner.

Pediatric neuroradiology is a specialized domain in itself; which calls for extensive knowledge to make a reasonable differential diagnosis. The disease entities are numerous; and with the advancement of genetic studies and molecular biology; the disease entities are acquiring a special dimension. Keeping oneself up-to date with the advances in pediatric neuroradiology is crucial for every pediatric neuroradiologist. The September issue of the symposium has dedicated sections on pediatric neuroimaging. The imaging in pediatric demyelinating and inflammatory diseases has been discussed in great detail by Dr Sudhakar and collegues [12, 13]. Pediatric demyelinating and inflammatory diseases are a complex group of disorders; the diagnoses of which are based on combined clinico-radiological and biochemical investigations. Pediatric radiologists and the physicians should have a clear idea of the disease subgroups and the imaging patterns. Similarly, pediatric stroke is not an uncommon disease entity. The causes are numerous; and calls for a detailed analysis of the imaging to rule out structural malformations and vascular causes. Dr Pruthi and colleagues have given us a detailed description of this complex entity in the September issue [14].

Neonates are not smaller children; and the diseases are much different in them. MR imaging in neonates faces various practical challenges; including the need for a prolonged sedation/ anesthesia; and susceptibility of the MR images to multiple artifacts. To interpret neonatal brain MR images; the radiologist need to be aware of the normal and abnormal myelination pattern in neonates; and various disease entities prevalent in them. Neonatal body MR imaging poses a greater challenge to the radiologist. Successful neonatal body MR imaging will require a high resolution small field of view imaging; yet being time efficient. Dr Shroff has described this emerging imaging technique in great detail in the September issue of the symposium [15]. Having wide experience in the field of pediatric neuroradiology for more than three decades in a busy children's hospital, he has elaborated the difficult topic with masterly ease.

To conclude, this symposium will be a ready reckoner to the radiologist and the practicing pediatrician both; in terms of the basics as well as advances in pediatric imaging.

\section{Compliance with Ethical Standards}

Conflict of Interest None.

Source of Funding None.

\section{References}

1. Jana M, Bhalla AS, Gupta AK. Approach to pediatric chest radiograph. Indian J Pediatr. 2016. doi:10.1007/s12098-015-1980-3.

2. Gajarajulu V, Natarajan B, Muralinath S. The radiograph of the pelvis as a window to skeletal dysplasias. Indian J Pediatr. 2016. doi:10.1007/s12098-015-1919-8.

3. Saxena AK, Gupta P, Sodhi KS. Ultrasonography: applications in pediatric abdomen. Indian J Pediatr. 2016. doi:10.1007/s12098015-1968-Z.

4. Practice guideline for the performance of an ultrasound examination of the neonatal spine. American Institute of Ultrasound in Medicine (AIUM). 2007; updated 2012.

5. Karnik AS, Karnik A, Joshi A. Ultrasound examination of pediatric musculoskeletal diseases and neonatal spine. Indian J Pediatr. 2016. doi:10.1007/s12098-015-1957-2.

6. Shekdar K. Imaging of abusive trauma. Indian J Pediatr. 2016. doi: 10.1007/s12098-016-2043-0.

7. Irodi A, Leena RV, Prabhu SM, Gibikote S. Role of computed tomography in pediatric chest conditions. Indian J Pediatr. 2016. doi:10.1007/s12098-015-1955-4.

8. Eapen A, Gibikote S. Role of computed tomography in pediatric abdominal conditions. Indian J Pediatr. 2016. doi:10.1007/s12098016-2030-5.

9. Kandasamy D, Goyal A, Sharma R, Gupta AK. Pediatric body magnetic resonance imaging. Indian J Pediatr. 2016. doi:10.1007/ s12098-015-1978-x.

10. Kandasamy D, Gamanagatti S, Gupta AK. Pediatric interventional radiology: vascular interventions. Indian J Pediatr. 2016. doi:10. 1007/s12098-016-2055-9.

11. Kandasamy D, Gamanagatti S, Gupta AK. Pediatric interventional radiology: non-vascular interventions. Indian J Pediatr. 2016. doi: 10.1007/s12098-015-1987-9.

12. Sudhakar SV, Muthusamy K, Mani S, Gibikote S, Shroff M. Imaging in pediatric demyelinating and inflammatory diseases of the brain- part 1. Indian J Pediatr. 2015. doi:10.1007/s12098-0151916-y.

13. Sudhakar SV, Muthusamy K, Mani S, Gibikote S, Shroff M. Imaging in pediatric demyelinating and inflammatory diseases of the brain- part 2. Indian J Pediatr. 2016. doi:10.1007/s12098-0162052-z.

14. Bhatia A, Pruthi S. Imaging of pediatric stroke. Indian J Pediatr. 2016. doi:10.1007/s12098-015-1982-1.

15. Krishnan $P$, Shroff M. Neuroimaging in neonatal hypoxic ischemic encephalopathy. Indian J Pediatr. 2016. doi:10.1007/s12098-0162042-1. 